\title{
Elastic-plastic-brittle transitions of potassium dihydrogen phosphate crystals: characterization by nanoindentation
}

\author{
Yong Zhang ${ }^{1} \cdot$ Ning $\mathrm{Hou}^{2} \cdot$ Liang-Chi Zhang ${ }^{3} \cdot$ Qi Wang ${ }^{1}$
}

Received: 19 May 2020/Revised: 7 June 2020/ Accepted: 23 July 2020/Published online: 2 September 2020

(C) The Author(s) 2020

\begin{abstract}
Potassium dihydrogen phosphate (KDP) crystals are widely used in laser ignition facilities as optical switching and frequency conversion components. These crystals are soft, brittle, and sensitive to external conditions (e.g., humidity, temperature, and applied stress). Hence, conventional characterization methods, such as transmission electron microscopy, cannot be used to study the mechanisms of material deformation. Nevertheless, understanding the mechanism of plastic-brittle transition in KDP crystals is important to prevent the fracture damage during the machining process. This study explores the plastic deformation and brittle fracture mechanisms of KDP crystals through nanoindentation experiments and theoretical calculations. The results show that dislocation nucleation and propagation are the main mechanisms of plastic deformation in KDP crystals, and dislocation pileup leads to brittle fracture during nanoindentation. Nanoindentation experiments using various indenters indicate that the external stress fields influence the plastic deformation of KDP crystals, and plastic deformation and brittle fracture are related to the material's anisotropy. However, the
\end{abstract}

Ning Hou

13b908074@hit.edu.cn

$\triangle$ Liang-Chi Zhang

liangchi.zhang@unsw.edu.au

1 School of Mechatronics Engineering, Harbin Institute of Technology, Harbin 150001, People's Republic of China

2 School of Mechatronics Engineering, Shenyang Aerospace University, Shenyang 110136, People's Republic of China

3 Laboratory for Precision and Nano Processing Technologies, School of Mechanical and Manufacturing Engineering, The University of New South Wales, Sydney, NSW 2052, Australia effect of loading rate on the KDP crystal deformation is practically negligible. The results of this research provide important information on reducing machining-induced damage and further improving the optical performance of KDP crystal components.

Keywords Potassium dihydrogen phosphate (KDP) crystal · Transition mechanism - Plastic deformation . Brittle fracture

\section{Introduction}

Potassium dihydrogen phosphate (KDP) crystals are crucial to laser ignition facilities as optical switching and frequency conversion components for inertial confinement fusion [1]. To fabricate the KDP crystal component, the main technology employed is ultra-precision diamond fly cutting $[2,3]$. However, machining-induced damage, such as crack and collapse, often emerges on the component surface/subsurface because of the brittleness of KDP crystals, significantly reducing the laser damage threshold of its components. To obtain a damage-free surface, KDP crystals should be machined in plastic mode. To date, however, few attempts have been made to understand the plastic-brittle transition behavior of KDP crystals. This limited understanding affects our ability to improve the surface quality of KDP crystals, restricting the optical performance of KDP devices.

Indentation experiments have been performed to investigate crack initiation in the brittle fracture of KDP crystals. Joshi et al. [4] conducted indentation experiments on KDP crystals with a Vickers tester and found that cracks appeared around the indentation on the KDP (100) plane when the load exceeded $50 \mathrm{~g}$. Cracks were also observed 
on the KDP (101) plane when the load was $70 \mathrm{~g}$. Reference [5] also indicated that at the $10 \mathrm{~g}$ load, cracks initiated on the (100) plane of KDP crystals, when a similar Vickers tester was used. Fang and Lambropoulos [6] measured the crack size on this plane with a Vickers indentation and reported that straight radial cracks emanated from the indentation corners when the load exceeded $0.25 \mathrm{~N}$. The above findings indicate that the magnitudes of the critical load necessary to produce cracks on KDP crystals vary among indentation experiments. As a result, determining the critical load through indentation experiments is difficult because the applied loads cannot be changed continually with the indentation method. By contrast, scratch testing with a continuous load is an alternative method that is used to investigate the critical load of the plastic-brittle transition of KDP crystals. For example, Cao et al. [7] conducted scratch testing with a spherical indenter on the (001) plane of KDP crystals and reported that brittle fractures appeared when the radial forces were 2940 and $3626 \mathrm{mN}$ along the [100] and [110] directions, respectively. Wang et al. [8,9] used a spherical indenter to implement nanoscratch testing with a continuous load. These researchers found that the critical loads of plastic-brittle transition were 104.5, 79.3 and $56.3 \mathrm{mN}$ along the $0^{\circ}, 45^{\circ}$ and $90^{\circ}$ directions on the second harmonic generation plane of KDP crystals, respectively [8]. On the third harmonic generation plane, the critical loads of brittle-plastic transition were 20.4, 33.6 and $63.2 \mathrm{mN}$ along the $0^{\circ}, 45^{\circ}$ and $90^{\circ}$ directions, respectively [9]. These results indicate that the brittle property of KDP crystals is anisotropic and sensitive to external stresses. Currently, the observation of experimentally generated cracks is a common method employed to determine the critical load of the plastic-brittle transition. However, the critical load obtained by scratch testing considerably differs from that observed in practice. Hence, at this point, such results cannot be directly used to deal with the machining-induced damage in KDP crystals. Therefore, to fully understand the mechanism and critical stress threshold of the plastic-brittle transitions of KDP crystals, theoretical analysis and calculation are necessary.

The insight into the plastic deformation mechanism of KDP crystals is essential to understand the plastic-brittle transition mechanism. The capacity of materials to resist plastic deformation is reflected by their hardness; hence, in view of their microhardness and nanohardness, KDP crystals have attracted considerable interest. For example, when the applied loads vary from $10 \mathrm{~g}$ to $100 \mathrm{~g}$, the microhardness values of KDP crystals range $190-135 \mathrm{~kg} / \mathrm{mm}^{2}$ and $190-160 \mathrm{~kg} / \mathrm{mm}^{2}$ on the (100) and (001) planes, respectively $[5,10]$. Kucheyev et al. [11] reported that under a $1000 \mu \mathrm{N}$ load using a spherical indenter, the nanohardness values of KDP crystals were $1.6 \pm 0.2$ and $2.0 \pm 0.2 \mathrm{GPa}$ on the (100) and (001) planes, respectively. These findings suggest that plastic deformations in KDP crystals may be produced at a microscale or nanoscale. Pop-in events are also correlated with the plastic deformation of KDP crystals during nanoindentation [12, 13]. Kucheyev et al. [11] observed multiple pop-in events in the load-displacement curves of KDP crystals and showed that under the $1000 \mu \mathrm{N}$ peak force with a spherical indenter, the first pop-in events appeared at loads of $225 \pm 52$ and $566 \pm 87 \mu \mathrm{N}$ on the (100) and (001) planes, respectively. References [14, 15] reported that pop-in events also existed on the doubler and tripler planes of KDP crystals. However, the origin of the pop-in event mechanism, which affects our understanding of the plastic deformation and brittle fracture mechanisms of KDP crystals, remains vague. Moreover, the study of the plastic deformation mechanism of KDP crystals using conventional characterization methods, such as transmission electron microscopy, is difficult to implement because the materials are soft, prone to fracture, and sensitive to external conditions (e.g., humidity, temperature, and applied stress).

In this study, the plastic deformation of KDP crystals is first analyzed with the aid of pop-in events using nanoindentation experiments. Thereafter, the material transition mechanism from plastic deformation to brittle fracture is further investigated. This study provides additional insight into the control of the brittle damage of KDP crystals during the machining process.

\section{Experimental}

The KDP crystals provided by the State Key Laboratory of Crystal Materials, Shandong University (Jinan, China) were produced using rapid growth technology. The crystal has a tetragonal system structure with Mohs hardness, Poisson's ratio, density, melting temperature and thermal conductivity of $1.5,0.24,2.338 \mathrm{~g} / \mathrm{cm}^{3}, 250{ }^{\circ} \mathrm{C}$ and $2.0 \mathrm{~W} \cdot(\mathrm{m} \cdot \mathrm{K})^{-1}$, respectively. Nanoindentation experiments were conducted on the (001) plane of KDP crystals. During the cleavage process, the energy generated can be released to obtain a damage-free surface because KDP crystals are brittle. A schematic of the mechanical stress cleavage method is shown in Fig. 1 [16]. A piece of KDP crystal is cleaved into two parts parallel to the (001) plane subjected

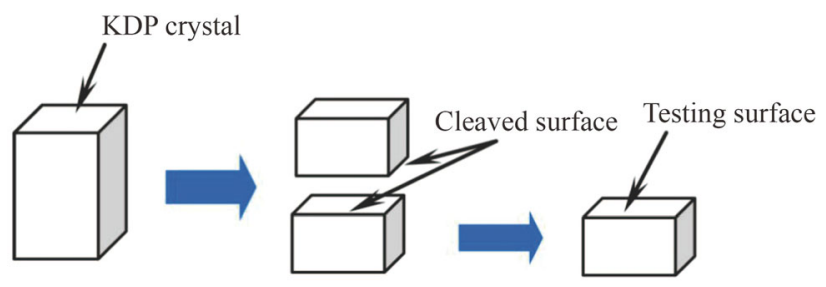

Fig. 1 Schematic of the mechanical stress cleavage method [16] 
to external stress, producing damage-free surfaces for nanoindentation experiments.

Nanoindentation experiments were conducted using a nanoindentation device (Triboindenter, Hysitron TI950, Hysitron Inc., Minneapolis, USA) at room temperature. The peak forces were set as 100, 500, 1000,3000 and $8000 \mu \mathrm{N}$, and the loading rates were $200 \mu \mathrm{N} / \mathrm{s}$ and 1600 $\mu \mathrm{N} \cdot \mathrm{s}^{-1}$. The Berkovich indenter (with an included angle of $142.35^{\circ}$ ) and conical indenters I and II (with apex angles of $60^{\circ}$ and $90^{\circ}$ and tip radii of $0.5 \mu \mathrm{m}$ and $1.22 \mu \mathrm{m}$, respectively) were used for the nanoindentation experiments. Each area function of the indenter was calibrated on a standard sample of fused quartz.

The projected area function of conical indenter $\mathrm{I}$ is precisely described as

$A=-\pi h^{2}+2 \pi r h$,

where $h$ is the indentation depth (nm).

The projected area function of conical indenter II is accurately expressed as

$A=-3.142 h^{2}+11175 h-143500 h^{0.5}+224470 h^{0.5}$.

The projected area function of the Berkovich indenter is correctly given as

$A=25.4 h^{2}+4043.1 h-69792 h^{0.5}+123430 h^{0.5}$.

\section{Results and discussion}

\subsection{Analysis and characterization of pop-in events}

The load-displacement curves of KDP crystals under peak forces of 500, 1000,3000 and $8000 \mu \mathrm{N}$ at a loading rate of $200 \mu \mathrm{N} / \mathrm{s}$ are shown in Fig. 2. To clearly observe the first pop-in events, the load-displacement curves at the initial stage are enlarged because pop-in events appear in each curve. A notable feature among the load-displacement curves is that the loads corresponding to the first pop-in events are practically identical. As shown in the inset in Fig. 2, the corresponding loads of the first pop-in events are $100,103,95$ and $94 \mu \mathrm{N}$ under peak forces of 500, 1000 , 3000 and $8000 \mu \mathrm{N}$, respectively. This means that the occurrence of the first pop-in events is independent of the peak force. Kucheyev et al. [11] reported that the loads corresponding to the first pop-in events were $566 \pm 87 \mu \mathrm{N}$ and $225 \pm 52 \mu \mathrm{N}$ on the (001) and (100) planes of KDP crystals using a spherical indenter, respectively. Zhang et al. [16] reported that in forming an indentation on the (210) plane, the corresponding load of the first pop-in event was also independent of the peak force. Therefore, the relationship between the first pop-in event and peak force found in this study is consistent with the published report. Moreover, the differences among the results of various studies with respect to the corresponding loads of the first pop-in event may be attributed to several factors, such as indenter shape, anisotropy of KDP crystals, and sample preparation process.

To understand the physics behind the first pop-in events in Fig. 2, the load-displacement curves corresponding to the elastic and elastic-plastic deformations during the nanoindentation of KDP crystals were calculated based on contact mechanics [17]. At the beginning of nanoindentation, the load-displacement curve of an ideal elastic deformation can be described as

$F=\frac{4}{3} E^{*} R^{1 / 2} h^{3 / 2}$,

where $F$ is the load $(\mu \mathrm{N}), R$ the indenter radius $(\mu \mathrm{m})$, and $E^{*}$ the elastic modulus of the indentation system (GPa) described as

$\frac{1}{E^{*}}=\frac{1-v_{\mathrm{m}}^{2}}{E_{\mathrm{m}}}+\frac{1-v_{\mathrm{i}}^{2}}{E_{\mathrm{i}}}$,

where $v_{\mathrm{m}}$ and $v_{\mathrm{i}}$ are the Poisson's ratios of the sample and indenter, respectively; $E_{\mathrm{m}}$ and $E_{\mathrm{i}}$ are the elastic moduli (GPa) of the sample and indenter, respectively.

References [17, 18] reported that the total depth $(\delta)$ penetrated by a spherical indenter into an elastic half-space was given as

$\delta=Q k$,

where

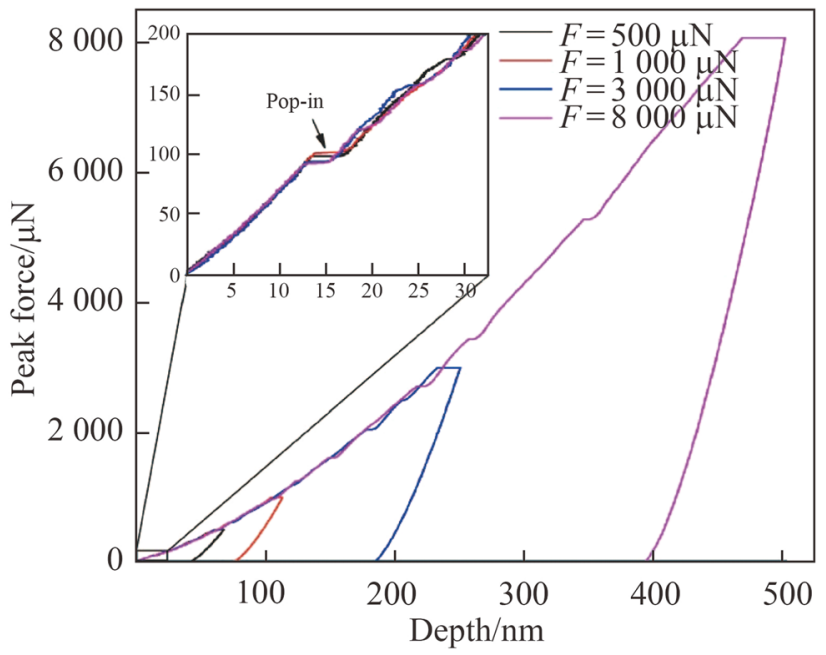

Fig. 2 Load-displacement curves under various peak forces with a conical indenter II 
$Q=\left(\frac{9}{16}\right)^{1 / 3}\left(\frac{F}{E^{*}}\right)^{2 / 3}$

and

$k=\left(\frac{1}{R}\right)^{1 / 3}$,

where $1 / R$ is the contact surface curvature.

The relationship between the depth $(\delta)$ and contact circular arc radius ( $a$ ) may be approximately described as

$\delta=\frac{a^{2}}{R}$.

The mean pressure $\left(P_{\mathrm{m}}\right)$ exerted on the contact circle is

$P_{\mathrm{m}}=\frac{F}{A}=\frac{F}{\pi a^{2}}$.

References [18, 19] reported that plastic deformation occurred when $P_{\mathrm{m}} / Y$ reached 3.0, where $Y$ is the yield stress of the material $(\mathrm{GPa})$.

Using Eqs. (6)-(10), the critical load under which the plastic deformation of a material begins can be calculated as

$F=\frac{9}{16}\left(\frac{R}{E^{*}}\right)^{2}(3 \pi Y)^{3}$.

Figure 3 shows the elastic and elastic-plastic deformation curves of KDP crystals calculated according to Eqs. (4) and (11) under a $500 \mu \mathrm{N}$ peak force. The $E_{\mathrm{m}}$ and $v_{\mathrm{m}}$ are $52.8 \pm 3.8 \mathrm{GPa}$ and 0.24 , respectively [12]; $Y$ is obtained from Ref. [20]. The $E_{\mathrm{i}}$ and $v_{\mathrm{i}}$ of the diamond indenter are $1050 \mathrm{GPa}$ and 0.1 , respectively [21].

After the KDP crystals undergo plastic deformation, the curve slopes between the elastic and elastic-plastic deformations are found to differ. Therefore, the yield point of

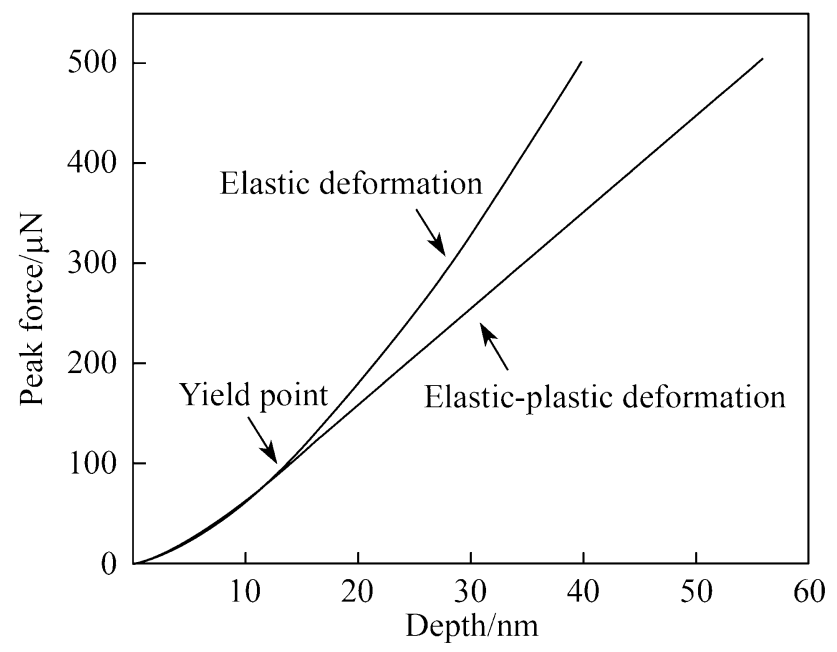

Fig. 3 Calculated load-displacement curves the elastic-plastic deformation can be determined by comparing its load-displacement curve to that of the elastic deformation, as shown in Fig. 3. The critical load corresponding to the yield point is approximately $100 \mu \mathrm{N}$, which considerably approximates the load of the first pop-in event, as shown in Fig. 2. This means that the first pop-in event corresponds to the yield point of KDP crystals, and after the first pop-in event, the KDP crystals will undergo plastic deformation during nanoindentation.

To analyze the influence of the first pop-in event on the plastic deformation of KDP crystals during nanoindentation, the load-displacement curves with peak forces of 100 , 500, 1000,3000 and $8000 \mu \mathrm{N}$ were compared with the ideal elastic deformation curves calculated by Eq. (4). Figure $4 \mathrm{a}$ shows the load-displacement curves under a 100 $\mu \mathrm{N}$ peak force. No pop-in event appears in the loading curve, and the slopes of the loading, unloading, and ideal elastic curves are considerably close. This indicates that KDP crystals only undergo elastic deformation under a peak force of $100 \mu \mathrm{N}$, which is consistent with the yield load observed in Fig. 3. In contrast to the conical indenter used in this study, Zhang et al. [16] utilized a Berkovich indenter to conduct nanoindentation tests on the KDP crystal (210) plane with a $100 \mu \mathrm{N}$ peak force. They also reported that the KDP crystals only underwent elastic deformation under this force.

To observe the pop-in events in the loading curve, the peak forces were increased to 500, 1000,3000 and 8000 $\mu \mathrm{N}$. Four pop-in events appear in the loading curves, of which the critical load of the first pop-in event is approximately $100 \mu \mathrm{N}$, as shown in Fig. 4b. When the load is less than $100 \mu \mathrm{N}$, the slope of the loading curve approximates that of the ideal elastic deformation curve; however, when the load exceeds $100 \mu \mathrm{N}$, the slope of the former is less than that of the latter. These results imply that the KDP crystal deformation changes from elastic to plastic when the load reaches $100 \mu \mathrm{N}$. The loads at which the deformation transitions from elastic to plastic are 103, 95 and $94 \mu \mathrm{N}$ under peak forces of 1000,3000 and $8000 \mu \mathrm{N}$ as shown in Figs. 4c-e, respectively. Moreover, with peak forces of $3000 \mu \mathrm{N}$ and $8000 \mu \mathrm{N}$, no cracks occur around the indentation, as shown in Fig. 5. Hence, these results indicate the deformation of KDP crystals during nanoindentation transitions from elastic to plastic at the first popin event. The yield load corresponding to the first pop-in event that generates plastic deformation is approximately $98 \pm 5 \mu \mathrm{N}$. Figure 5 also shows the indentation morphology under the two peak forces; however, these marks do not well match the shape of the indenter because of the anisotropy of KDP crystals.

To further understand the plastic deformation of KDP crystals, the stress generated during loading was analyzed according to Eqs. (2) and (10), as shown in Fig. 6. The 


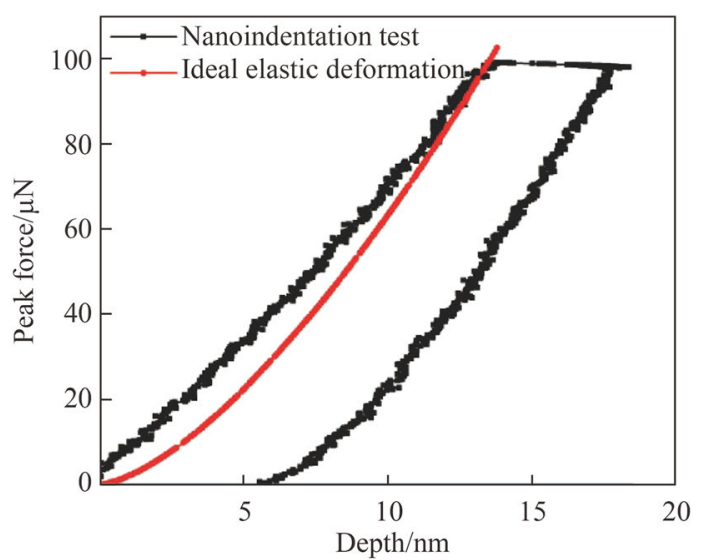

(a) Peak force of $100 \mu \mathrm{N}$

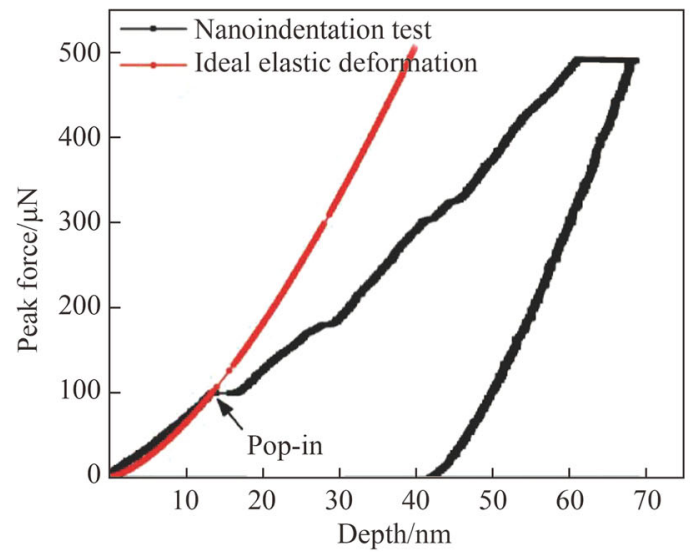

(b) Peak force of $500 \mu \mathrm{N}$

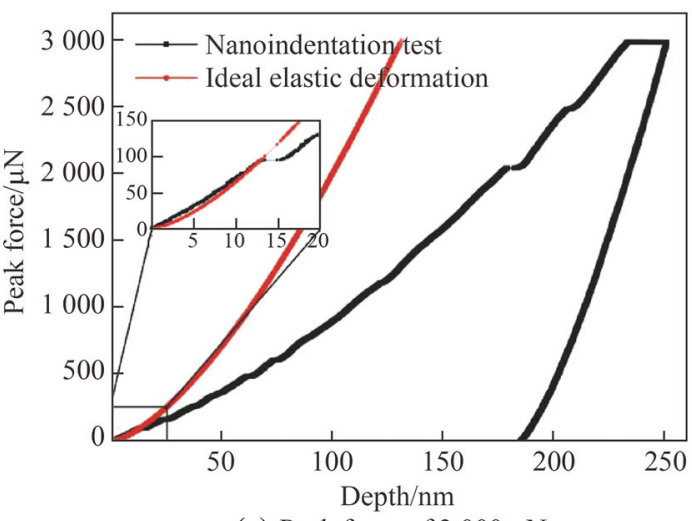

(e) Peak force of $3000 \mu \mathrm{N}$

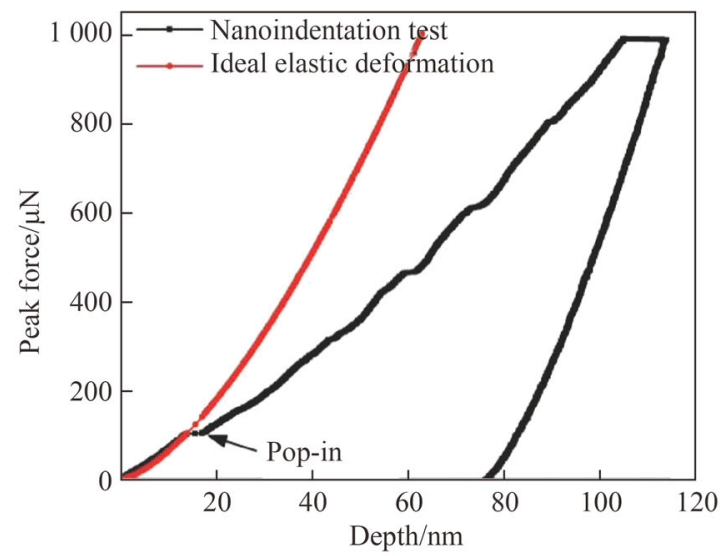

(c) Peak force of $1000 \mu \mathrm{N}$

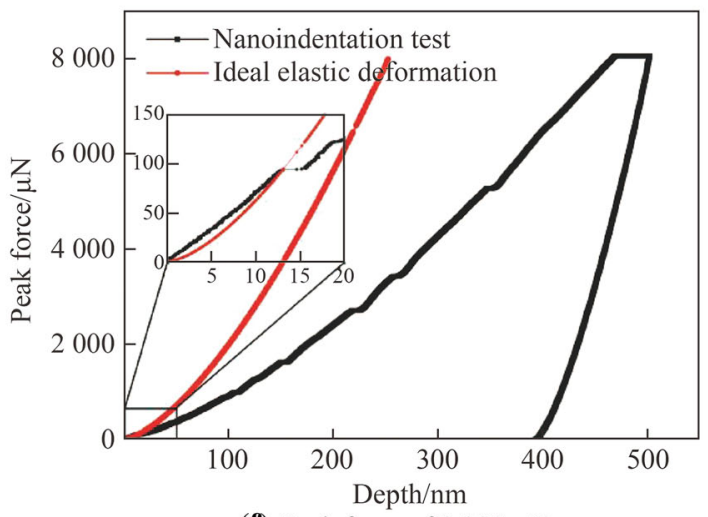

(f) Peak force of $8000 \mu \mathrm{N}$

Fig. 4 Tested and calculated load-displacement curves under various peak forces with a conical indenter II

loading curves of the KDP crystals are divided into three stages.

The elastic stage is observed when the stress is less than the yield point. The loading curves overlap because the KDP crystal only undergoes elastic deformation under a peak force of $100 \mu \mathrm{N}$, as shown in Fig. 4a.

The yield stage occurs at the yield point; the stress is constant, but the indentation depth continuously increases. The yield stress in KDP crystals is approximately $1.92 \pm$
0.05 GPa during the nanoindentation using conical indenter II.

The plastic stage is observed when the stress, which increases with the indentation depth, exceeds the yield point. At $2.85 \mathrm{GPa}$, however, the stress decreases with increasing indentation depth. Then, as the indentation depth further increases, the stress increases again, as shown in Fig. 6b. As a result, the relationship between stress and depth in the plastic stage at room temperature is nonlinear. 


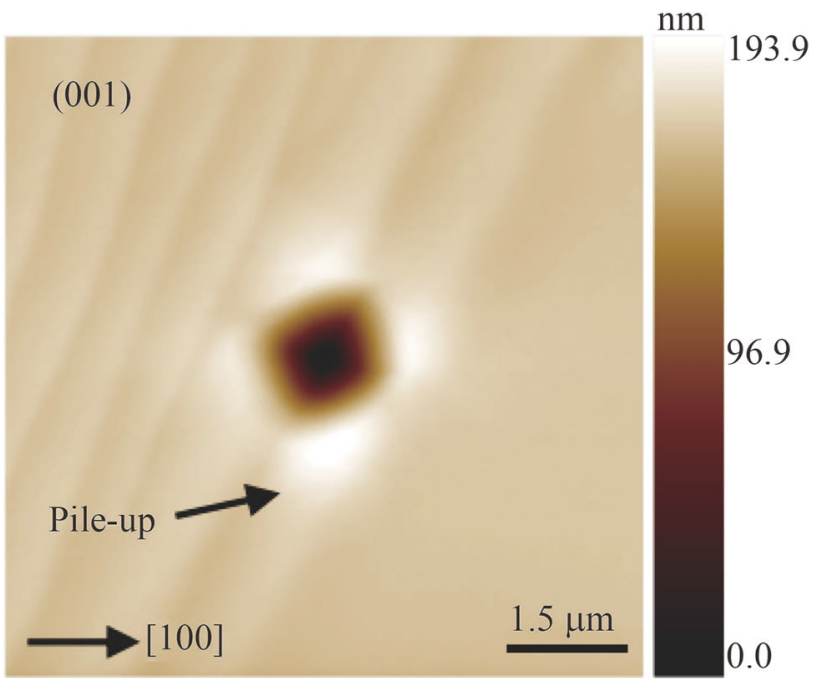

(a) Peak force of $3000 \mu \mathrm{N}$

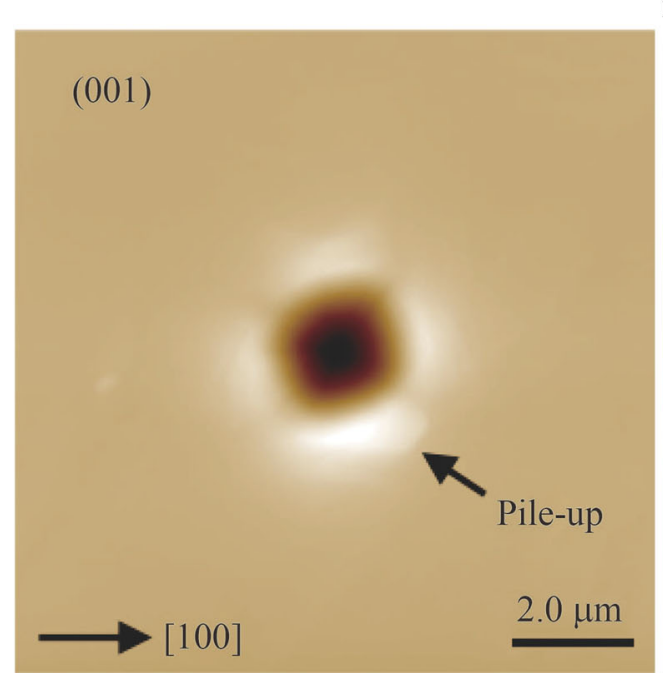

$\mathrm{nm}$ 454.5

(b) Peak force of $8000 \mu \mathrm{N}$

Fig. 5 Indentation morphology under various peak forces with a conical indenter II

\subsection{Formation mechanism of plastic deformation during loading}

Based on the above analysis, it is concluded that the KDP crystal begins to undergo plastic deformation at the first pop-in event during nanoindentation. This section presents the investigation of the plastic deformation mechanism in KDP crystals by examining the first pop-in event based on the theory of dislocation nucleation. It should be noted that the critical stress at which the KDP crystals undergo phase transition at room temperature is $7.5 \mathrm{GPa}$ [22]. Therefore, because the $1.92 \mathrm{GPa}$ yield stress is less than $7.5 \mathrm{GPa}$, the plastic deformation mechanism of KDP crystals is not the phase transition during nanoindentation. Furthermore, phase transition in the surface/subsurface was not detected

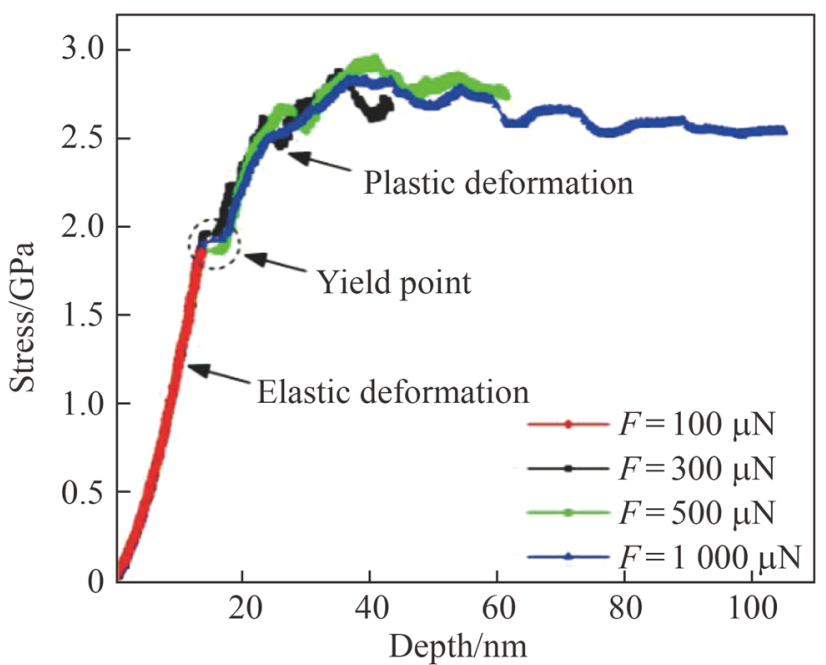

(a) Peak forces from $100 \mu \mathrm{N}$ to $1000 \mu \mathrm{N}$

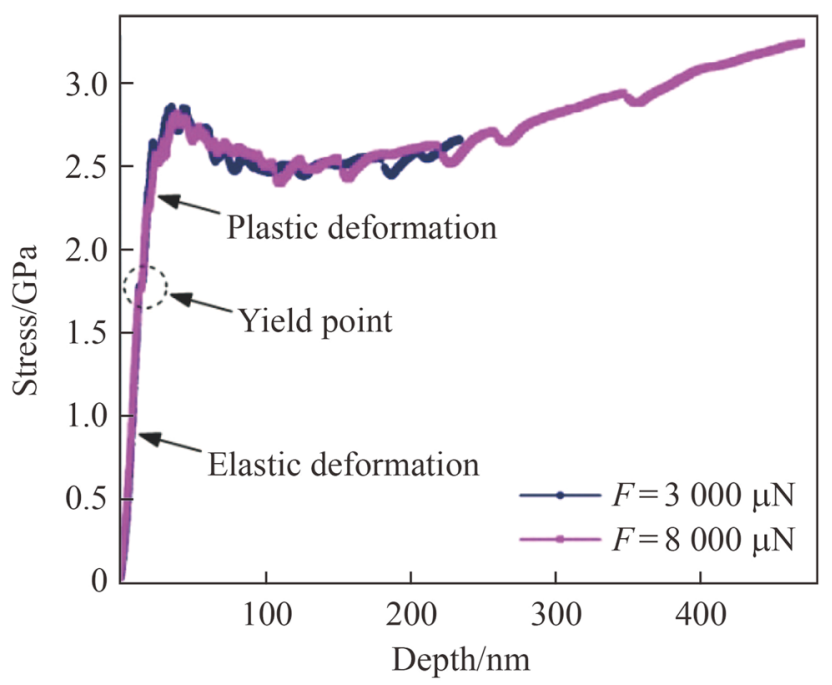

(b) Peak forces $3000 \mu \mathrm{N}$ and $8000 \mu \mathrm{N}$

Fig. 6 Stress-displacement curves under different peak forces

by the grazing incidence X-ray diffraction method after the mechanical processing of KDP crystals [23].

To explain the plastic deformation mechanism of KDP crystals during nanoindentation, the external stress is calculated with the aid of the theory of dislocation nucleation. The elastic energy $\left(E_{\mathrm{e}}\right)$ of a dislocation loop with a Burgers vector $(b)$ and radius $(r)$ can be described as [24]

$E_{\mathrm{e}}=\frac{G b^{2} r}{4} \frac{2-v}{1-v}\left(\ln \frac{4 r}{r_{\mathrm{o}}}-2\right)$,

where $v$ is Poisson's ratio, $r_{\mathrm{o}}$ the radius of dislocation nucleation (nm), and $G$ the shear modulus (GPa).

The strain energy $\left(E_{\tau}\right)$ released during the formation of a dislocation loop under a resolved shear stress $(\tau)$ is [24]

$E_{\tau}=-\tau b \pi r^{2}$. 
The maximum total energy $\left(E_{\mathrm{e}}+E_{\tau}\right)$ provides the maximum free energy $\left(E_{\mathrm{c}}\right)$ necessary to form a dislocation loop. For $E_{\mathrm{c}}=0$, the critical stress $\left(\tau_{\mathrm{c}}\right)$ of dislocation nucleation is obtained as

$\tau_{\mathrm{c}}=\frac{G b}{\pi e^{3} r_{\mathrm{o}}} \frac{2-v}{1-v}$

where $e$ is the Euler number (i.e., 2.7).

As a result of the anisotropic mechanical property of the KDP crystal, the $b, r_{\mathrm{o}}$, and $G$ are $0.63 \mathrm{~nm}, b / 3$, and 19.8-22.8 GPa on the (001) plane, respectively. Hence, the critical stress of dislocation nucleation in the KDP crystal is in the range 2.23-2.56 GPa, as calculated by Eq.(14). The calculated average stress value approximates the experimental yield stress of $1.92 \mathrm{GPa}$, which generates the first pop-in event, as shown in Fig. 6a. This means that dislocation nucleation and propagation are the main mechanisms of the plastic deformation of KDP crystals during nanoindentation. When the critical stress of dislocation nucleation is calculated based on the theory, its magnitude exceeds the experimental yield stress because the KDP crystal is assumed to be an integrated lattice without defects. However, in the nanoindentation experiments, such defects inevitably reduce the intrinsic strength of KDP crystals [25]. These are similar to the dislocation etch pits that Guin et al. [20] observed after the KDP crystals are indented at elevated temperatures and they also found that the crystals had five slip systems. The foregoing is consistent with the observation in this study, i.e., the KDP crystals plastically deform through the dislocation motion at room temperature during nanoindentation. Kucheyev et al. [11] and Peng et al. [12] also inferred that the occurrence of pop-in events was governed by the dislocation motion during the nanoindentation of KDP crystals.

The elucidation on the plastic deformation mechanism in KDP crystals is extremely important to understand the mechanical behavior of the material during nanoindentation, as shown in Fig. 6. Typically, the variation in atomic distance controls the elastic deformation of KDP crystals. Atoms return to their equilibrium positions when the external load is removed. When the external load exceeds the critical stress of dislocation nucleation, the KDP crystal begins to yield. At the yield stage, although the applied stress remains unchanged, the deformation continues. After the yield stage, the stress increases with the indentation depth because more slip systems are activated. When all slip systems are active, the stress reaches the maximum. In this case, the deformation of KDP crystals facilely occurs along different slip systems as the indentation depth increases. However, when the number of dislocations reaches a certain quantity, the dislocation interaction increases the resistance to deformation, explaining the increase in stress when the indentation depth exceeds 100 $\mathrm{nm}$, as shown in Fig. $6 \mathrm{~b}$.

\subsection{Effects of loading rate and indenter shape on plastic deformation}

The stress-displacement curves at loading rates of $200 \mu \mathrm{N} / \mathrm{s}$ and $1600 \mu \mathrm{N} / \mathrm{s}$ under a $1000 \mu \mathrm{N}$ peak force are shown in Fig. 7a. The two curves practically overlap, indicating that the effect of loading rate on the plastic deformation of KDP crystals is negligible during nanoindentation. This is consistent with the influence of the loading rate on the critical load of the first pop-in event reported in Ref. [16]. However, the stress-displacement curves between the Berkovich indenter and conical indenter II differ, as shown in Fig. 7b. When the indentation depth is the same, the applied stress

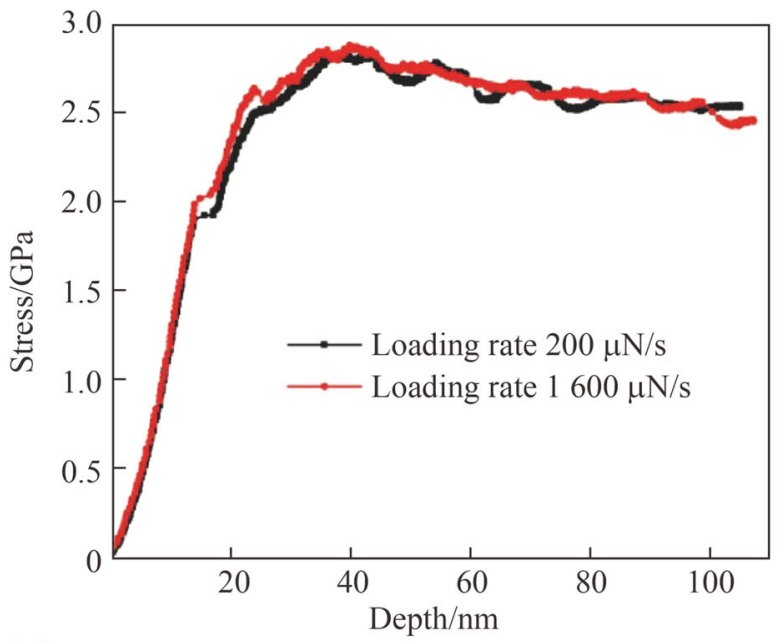

(a) Effect of loading rate on plastic deformation under a peak force of $1000 \mu \mathrm{N}$ with a conical indenter II

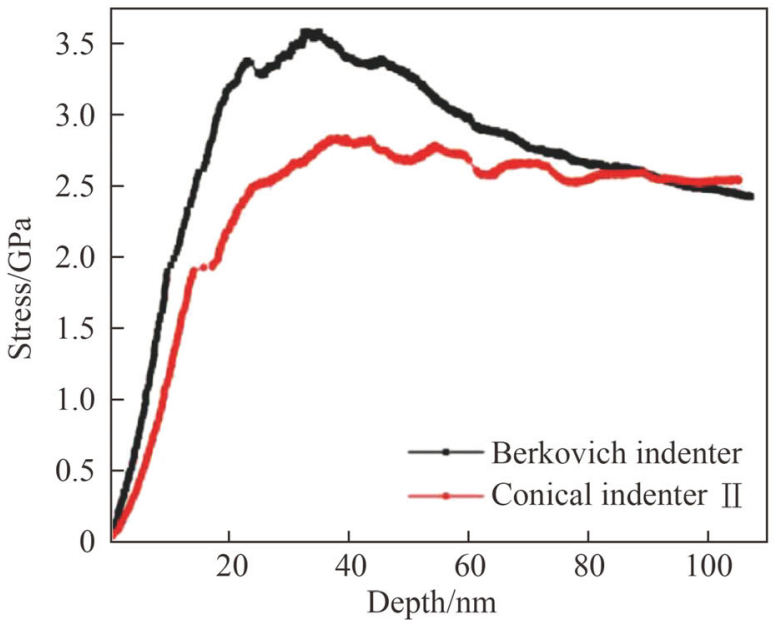

(b) Effect of indenter shape on plastic deformation with a peak force of $1000 \mu \mathrm{N}$ and loading rate of $200 \mu \mathrm{N} / \mathrm{s}$

Fig. 7 Stress-displacement curves during loading 
using the Berkovich indenter exceeds that using conical indenter II, implying that it is more difficult to initiate the elastic-plastic deformation of KDP crystals through the former than through the latter. Section 3.2 explains that this plastic deformation is mainly controlled by the dislocation motion. Hence, the higher the applied stress, the higher the dislocation density; consequently, the dislocation interaction hinders further plastic deformation. Therefore, the external stress fields significantly influence the plastic deformation of KDP crystals.

\subsection{Transition mechanism from plastic deformation to brittle fracture}

The plastic deformation mechanism of KDP crystals during nanoindentation is primarily the dislocation motion, which can be limited by the dislocation interaction, obstacles, and crystal lattice [26]. As a result, dislocation pileups occur, causing stress concentrations; the dislocation pileup schematic is shown in Fig. 8. After the dislocations move along the slip plane, the dislocation pileups appear at point $O$. The stress $(\sigma)$ generated in front of the piled-up group of dislocations is expressed as [27]

$\sigma=\sigma_{\mathrm{s}} \sqrt{\frac{L}{r}} f(\theta)$,

where $L$ is the distance along the slip plane that the dislocation moves $(\mathrm{nm})$, and $r$ is the distance along the $O P$ direction $(\mathrm{nm})$.

The shear stress $\left(\sigma_{\mathrm{s}}\right)$ along the slip plane is

$\sigma_{\mathrm{s}}=\sqrt{\frac{3 \pi \gamma G}{8(1-v) L}}$

where $\gamma$ is the fracture surface energy.

The function of the indenter apex angle $(f(\theta))$ is denoted as

$f(\theta)=\sin \theta \cos \frac{\theta}{2}$.

Hence, Eq. (15) can be rewritten as

$\sigma=\sqrt{\frac{3 \pi \gamma G}{8 r(1-v)}} \sin \theta \cos \frac{\theta}{2}$.

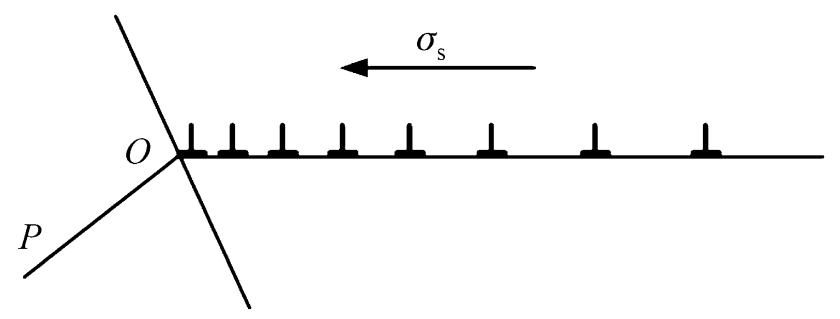

Fig. 8 Schematic of dislocation pileup
For the KDP crystal, the $\gamma$ is approximately $6.5 \mathrm{~J} / \mathrm{m}^{2}$ [28], and the $r$ and indenter apex angle are set as $11 \mathrm{~nm}$ and $60^{\circ}$, respectively. The magnitude of the stress generated in front of the piled-up dislocations is in the range 3.19-3.43 GPa because of the anisotropy indicated by the $G$ in Eq. (18).

To verify whether the stress induced by the dislocation pileup can lead to the brittle fracture of KDP crystals, nanoindentation was conducted using conical indenter I with an apex angle of $60^{\circ}$ and a tip radius of $0.5 \mu \mathrm{m}$. A "platform" appears when the load is $7136 \mu \mathrm{N}$, as shown in Fig. 9. The corresponding "platform" depth is $175 \mathrm{~nm}$, but that of the pop-in events is only $11 \mathrm{~nm}$. This means that the "platform" differs from the pop-in events and may be correlated to the brittle fracture. If the KDP crystals undergo brittle fracture at the "platform" position, then the deformation resistance will significantly decrease. In the stress-load curve shown in Fig. 10, the stress suddenly decreases at the $7136 \mu \mathrm{N}$ load and then increases to $3.15 \mathrm{GPa}$, indicating that the KDP crystals experience brittle fracture and lose their ability to resist deformation. The indentation morphology shown in Fig. 11 also confirms the occurrence of brittle fracture as evinced by the formation of some cracks around the indentation mark. It should be noted that the stress $(3.15 \mathrm{GPa})$ when the KDP crystals begin to undergo brittle fracture approximates that calculated in the front of the piled-up dislocations. This means that the dislocation pileup is the primary transition mechanism from plastic deformation to brittle fracture during nanoindentation.

The indentation mark is not consistent with the shape of conical indenter I, as shown in Fig. 11; this is attributed to the anisotropy of KDP crystals. The figure further shows that after unloading, the deformation of KDP crystals presents quadratic symmetry. This is because the

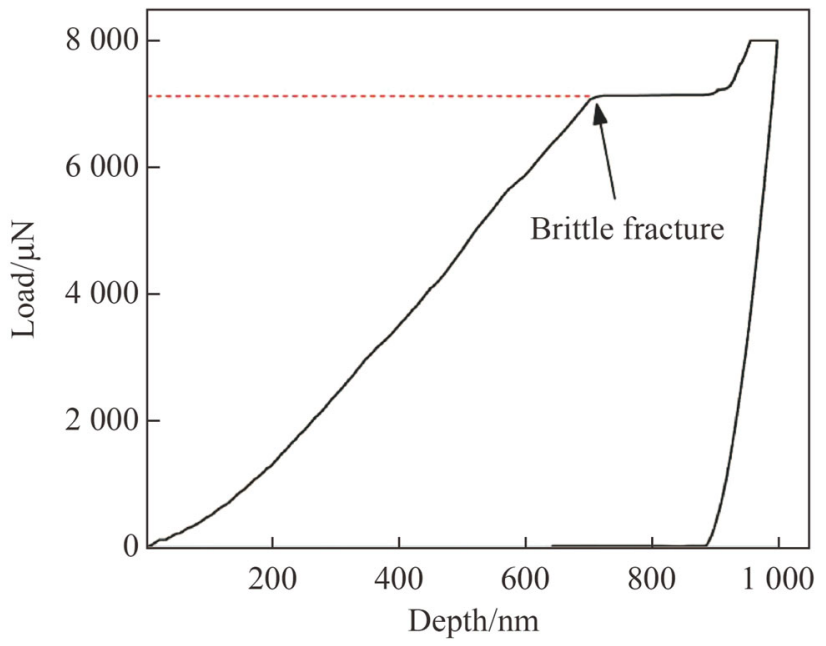

Fig. 9 Load-displacement curve during nanoindentation 


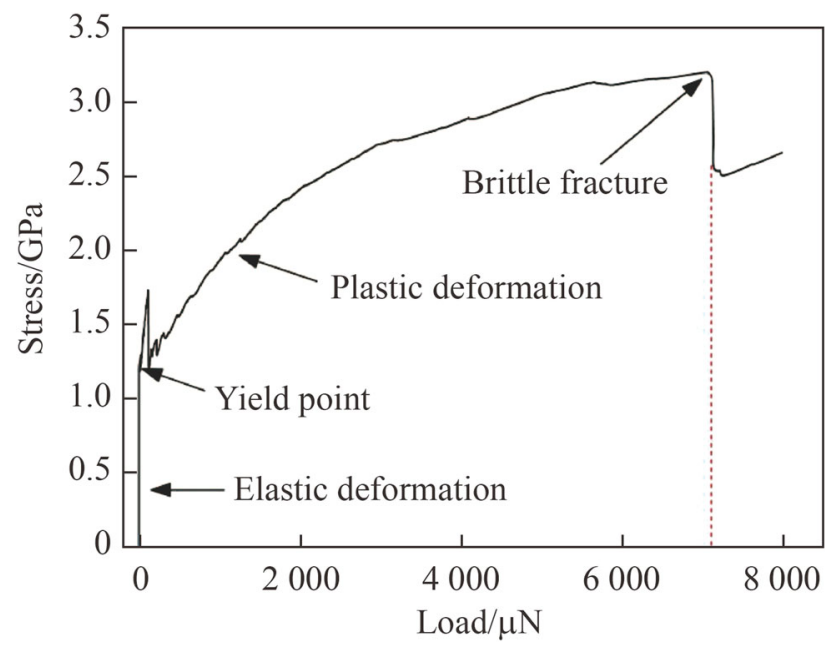

Fig. 10 Stress-load curve during loading

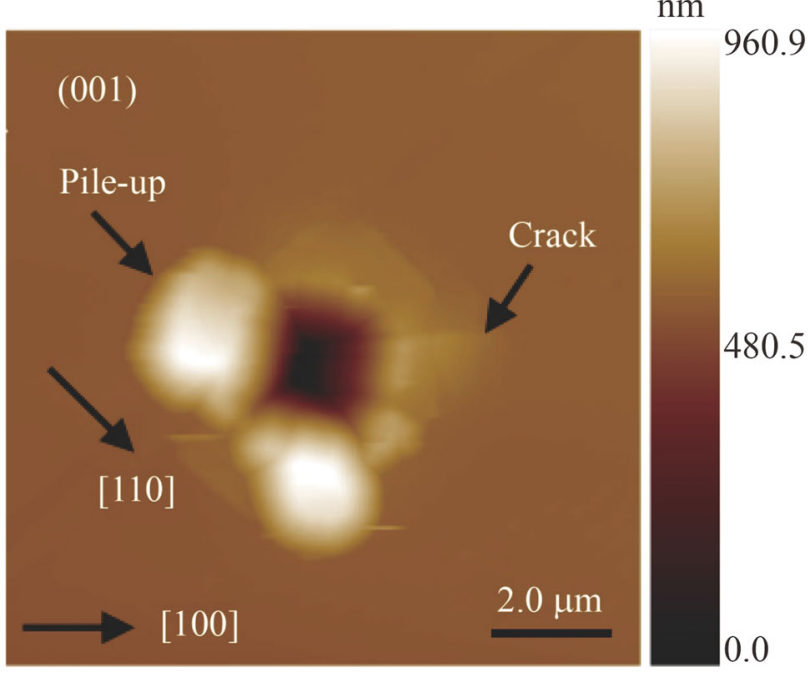

Fig. 11 Indentation morphology

microstructure of KDP crystals has quadratic symmetry on the (001) plane; this also leads to the quadratic symmetry of mechanical properties, such as elastic modulus and hardness. The elastic modulus of KDP crystals is minimal along the [110] direction on the (001) plane; accordingly, the material deformation along this direction is maximal, as shown in Fig. 11. Based on this result, it can be observed that the anisotropy of KDP crystals considerably influences the deformation behavior of crystals under the external force. If the brittle fracture of KDP crystals does not occur, the anisotropy of the material deformation will be evident after the conical indenter is unloaded because of the mutual restriction imposed by the materials. However, when the damage caused by cracking appears around the indentation on the KDP (001) plane, the internal stress distribution around the indentation is uneven. This further affects the anisotropy of the deformation behavior of KDP crystals, as shown in Figs. 5 and 11.

\section{Conclusions}

In this study, the brittle-plastic transition behavior of KDP crystals was investigated through nanoindentation. It is concluded that the deformation of KDP crystals transitions from elastic to plastic at the first pop-in event during nanoindentation, and the occurrence of the first pop-in event is independent of the peak force. During nanoindentation, the dislocation nucleation and propagation are the main mechanisms initiating the plastic deformation of KDP crystals; thereafter, the dislocation pileup is the primary transition mechanism from the plastic deformation to brittle fracture. The effect of loading rate on the plastic deformation of KDP crystals is negligible. The plastic deformation and brittle fracture of KDP crystals are related to the anisotropy of the material and external stress fields.

Acknowledgments This work was supported by the National Natural Science Foundation of China (NSFC) (Grant Nos. 51875137 and 51905356), the Natural Science Foundation of Heilongjiang Province (Grant No. E2018033), and the Australian Research Council (ARC) (Grant No. DP170100567). The authors thank Weidong Liu and Zhonghuai $\mathrm{Wu}$ for calculating the first pop-in event in this study.

Open Access This article is licensed under a Creative Commons Attribution 4.0 International License, which permits use, sharing, adaptation, distribution and reproduction in any medium or format, as long as you give appropriate credit to the original author(s) and the source, provide a link to the Creative Commons licence, and indicate if changes were made. The images or other third party material in this article are included in the article's Creative Commons licence, unless indicated otherwise in a credit line to the material. If material is not included in the article's Creative Commons licence and your intended use is not permitted by statutory regulation or exceeds the permitted use, you will need to obtain permission directly from the copyright holder. To view a copy of this licence, visit http://creativecommons. org/licenses/by/4.0/.

\section{References}

1. De Yoreo JJ, Burnham AK, Whitman PK (2002) Developing $\mathrm{KH}_{2} \mathrm{PO}_{4}$ and $\mathrm{KD}_{2} \mathrm{PO}_{4}$ crystals for the world's most power laser. Int Mater Rev 47(3):113-152

2. Chen G, Sun Y, An C et al (2018) Measurement and analysis for frequency domain error of ultra-precision spindle in a flycutting machine tool. Proc Inst Mech Eng Part B J Eng Manuf 232(9):1501-1507

3. Chen G, Sun Y, Zhang F et al (2017) Influence of ultra-precision fly cutting spindle error on surface frequency domain error formation. Int J Adv Manuf Technol 88(9/12):3233-3241

4. Joshi MS, Antony AV, Rao PM (1980) Microhardness investigations on potassium dihydrogen phosphate crystals. Cryst Res Technol 15(6):743-746 
5. Sengupta S, Sengupta SP (1992) Microhardness studies in gelgrown $\mathrm{ADP}$ and KDP single crystals. Bull Mater Sci 15(4):333-338

6. Fang T, Lambropoulos JC (2002) Microhardness and indentation fracture of potassium dihydrogen phosphate (KDP). J Am Ceram Soc 85(1):174-178

7. Cao XS, Wu DJ, Wang B et al (2008) Analysis on mechanical property of anisotropy of KDP crystal. J Synthetic Cryst 37(3):704-709

8. Wang D, Feng PF, Zhang CL et al (2012) Experimental research on the influence of the KDP crystal anisotropy on scratch characteristics. J Synthetic Cryst 41(3):568-572

9. Wang D, Feng PF, Zhang CL et al (2013) Experimental research on micro scale mechanics behavior of potassium dihydrogen phosphate crystal. Chin J Mech Eng 49(7):148-153

10. Rajesh NP, Kannan V, Raghavan PS et al (2002) Optical and microhardness studies of KDP crystals grown from aqueous solutions with organic additives. Mater Lett 52(4/5):326-328

11. Kucheyev SO, Siekhaus WJ, Land TA et al (2004) Mechanical response of $\mathrm{KD}_{2 x} \mathrm{H}_{2(1-x)} \mathrm{PO}_{4}$ crystals during nanoindentation. Appl Phys Lett 84(13):2274-2276

12. Peng J, Zhang LC, Lu XC (2014) Elastic-plastic deformation of KDP crystals under nanoindentation. Mater Sci Forum 773/774:705-711

13. Borc J, Sangwal K, Pritula I et al (2017) Investigation of pop-in events and indentation size effect on the (001) and (100) faces of KDP crystals by nanoindentation deformation. Mater Sci Eng A 708:1-10

14. Guo XG, Zhang XJ, Tang XZ et al (2013) Nanoindentation on the doubler plane of KDP single crystal. J Semicond 34(3):21-25

15. Lu C, Gao H, Wang J et al (2010) Mechanical properties of potassium dihydrogen phosphate single crystal by the nanoindentation technique. Mater Manuf Processes 25(8):740-748

16. Zhang Y, Zhang LC, Liu M et al (2016) Revealing the mechanical properties of potassium dihydrogen phosphate crystals by nanoindentation. J Mater Res 31(8):1056-1064

17. Johnson KL (1985) Contact mechanics. Cambridge University Press, Cambridge

18. Field JS, Swain MV (1993) A simple predictive model for spherical indentation. J Mater Res 8(2):297-306

19. Francis HA (1976) Phenomenological analysis of plastic spherical indentation. J Eng Mater Technol 98(3):272-281

20. Guin CH, Katrich MD, Savinkov AI et al (1980) Plastic strain and dislocation structure of the KDP group crystals. Cryst Res Technol 15(4):479-488

21. Chang L, Zhang L (2009) Mechanical behaviour characterisation of silicon and effect of loading rate on pop-in: a nanoindentation study under ultra-low loads. Mater Sci Eng A 506(1/2):125-129

22. Cai W, Katrusiak A (2013) Structure of the high-pressure phase IV of $\mathrm{KH}_{2} \mathrm{PO}_{4}$ (KDP). Dalton Trans 42(4):863-866

23. Hou N, Zhang Y, Zhang L et al (2016) Assessing microstructure changes in potassium dihydrogen phosphate crystals induced by mechanical stresses. Scr Mater 113:48-50

24. Leipner HS, Lorenz D, Zeckzer A et al (2001) Nanoindentation pop-in effect in semiconductors. Physica B 308/310:446-449

25. Wang B, Wang SL, Fang CS et al (2005) Effects of $\mathrm{Fe}^{3+}$ ion on the growth habit of KDP crystal. J Synthetic Cryst 34(2):205-208

26. Zhang Y, Hou N, Zhang LC (2018) Investigation into the room temperature creep-deformation of potassium dihydrogen phosphate crystals using nanoindentation. Adv Manuf 6(4):376-383

27. Stroh AN (1957) A theory of the fracture of metals. Adv Phys 6(24):418-465

28. Wiederhorn SM (1968) Fracture surface energy of glass. J Am Ceram Soc 52(2):99-105

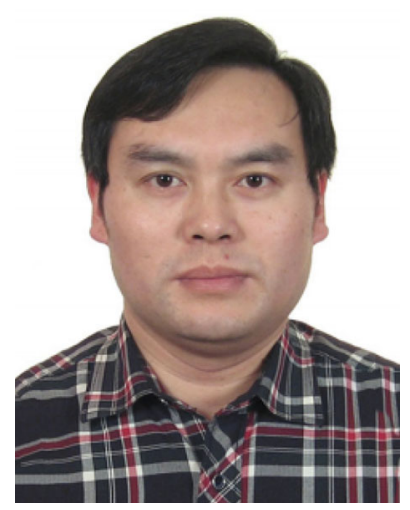

Yong Zhang is an associate professor and doctoral supervisor at Harbin Institute of Technology. Currently, he is engaged in the research of intelligent machining equipment and its process control technology, ultraprecision machining technology, subsurface damage mechanism during ultra-precision machining, and material mechanical behavior at micro/nano scale.

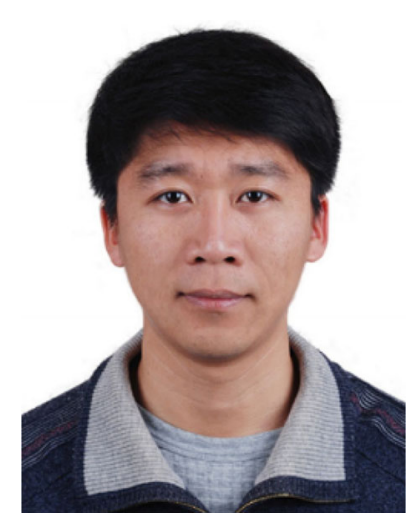

Ning Hou is a lecturer at Shenyang Aerospace University. His research focuses on the subsurface damage of KDP crystals and ultraprecision machining technology.

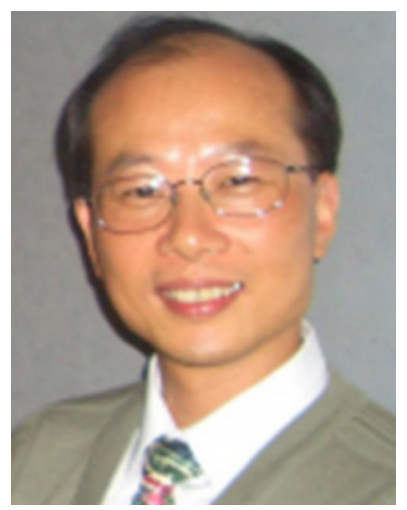

Liangchi Zhang (Scientia Professor) is from the University of New South Wales. His research interests include: precision manufacturing, bio-manufacturing, nanotechnology, characterisation of advanced materials, tribology, solid mechanics, computational mechanics.

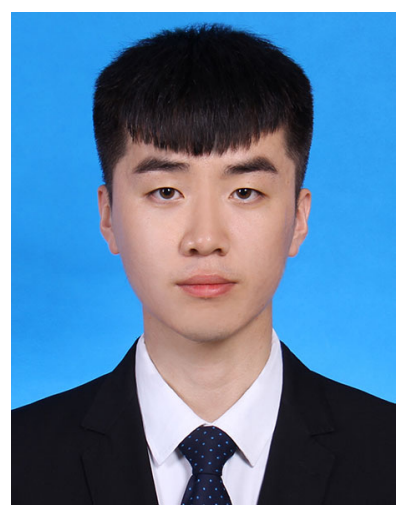

Qi Wang is a graduate student at Harbin Institute of technology. His research focuses on the ultraprecision machining technology of KDP crystals. 